\title{
Evaluation of the Differences Between Measurements in Multiple Institutions and Calculation Modeled by Representative Beam Data in Prostate VMAT Plan
}

\author{
HIRONAO GOTO ${ }^{1}$, HIROKAZU MIZUNO ${ }^{1}$, YUICHI AKINO ${ }^{2}$, MASARU ISONO ${ }^{3}$, YOSHIHIRO TANAKA ${ }^{4}$, \\ NORIHISA MASAI ${ }^{5}$, TOSHIJIRO YAMAMOTO ${ }^{6}$ and MASAHIKO KOIZUMI ${ }^{1}$ \\ ${ }^{1}$ Department of Medical Physics and Engineering, Osaka University Graduate School of Medicine, Osaka, Japan; \\ ${ }^{2}$ Division of Medical Physics, Osaka University Hospital Oncology Center, Osaka, Japan; \\ ${ }^{3}$ Department of Radiation Oncology, Osaka International Cancer Institute, Osaka, Japan; \\ ${ }^{4}$ Department of Radiation Therapy, Japanese Red Cross Society Kyoto Daiichi Hospital, Kyoto, Japan; \\ ${ }^{5}$ Miyakojima IGRT Clinic, Osaka, Japan; \\ ${ }^{6}$ Department of radiation therapy, Saiseikai Noe Hospital, Osaka, Japan
}

\begin{abstract}
Background/Aim: This study aimed to investigate the potential differences between multi-institutional measurements and treatment planning system (TPS) calculation modeled by representative beam data for patient-specific quality assurance (QA), including multi-leaf collimator (MLC) parameters. Materials and Methods: Eleven TrueBeam from nine institutions were used in this study. Volumetric arc therapy (VMAT) plan for verification was created using Eclipse. The point dose of the CC13 ionization chamber and the dose distribution of the GAFCHROMIC EBT3 film were measured and analyzed. Results: Point dose differences in patient-specific $Q A$ provided a mean \pm standard deviation of $1.0 \% \pm 0.6 \%$. Mean gamma pass rates of dose distribution were in excess of $99 \%$ and $96 \%$ for $3 \% / 2 \mathrm{~mm}$ and $2 \% / 2 \mathrm{~mm}$ gamma criteria, respectively. Conclusion: There was good agreement between measurements and calculations, indicating the small influence of complex VMAT in the underlying processes. Therefore, implementation of the same MLC parameters on TPS among different institutions with the same planning policy should be considered to ensure consistency and efficiency in radiation treatment processes.
\end{abstract}

This article is freely accessible online.

Correspondence to: Hirokazu Mizuno, Department of Medical Physics and Engineering Graduate School of Medicine, Osaka University. 1-7 Yamada-oka, Suita, Osaka, 565-0871, Japan. Tel: +81 668792564, Fax: +81668792564, e-mail: h_mizuno@radonc. med.osaka-u.ac.jp

Key Words: Treatment planning system, volumetric arc therapy, dose distribution, irradiation, MLC transmission, dosimetric leaf gap.
Novel radiotherapy devices are becoming increasingly accurate, and a prominent example involves TrueBeam (Varian Medical Systems, Inc., Palo Alto, CA) where the respective variance has become very narrow (1-4). Varian Medical Systems provide representative beam data (RBD) that is averaged beam data measured by three TrueBeam at the Duke University (1). It has been reported that the measured TrueBeam and the RBD data were very similar. Tanaka et al. (5) have reported that the averaged measurement data for treatment planning system (TPS) modeling indicated a small variance. More specifically, the percentage depth dose (PDD) difference between the RBD and the averaged measurement data was within $0.5 \%$, whereas the off-center ratio $(\mathrm{OCR})$ for a flat $10 \times 10,20 \times 20$, and $30 \times 30 \mathrm{~cm}^{2}$ region between the RBD and the averaged measurement data was within $1 \%$.

Multi-leaf collimator (MLC) systems enable the precise delivery of the intended dose according to planned dose constraints by complex MLC motion (6-9). The accuracy of the MLC position influences the intensity-modulated radiation therapy (IMRT) and the volumetric modulated arc therapy (VMAT) (10-12). Simultaneously, MLC parameters in TPS need to be modified to maintain consistency with patient-specific quality assurance (QA) measured data (11, 13). In Eclipse (Varian Medical Systems), MLC parameters are controlled by the dosimetric leaf gap (DLG) and the transmission factor, which are not provided as RBD (14). Moreover, the optimum MLC parameters depend on the plan, delivery system, TPS, and tools used in patient-specific QA $(12,13,15)$. Our previous report suggests that the variation between the optimum and the measured MLC parameters was significantly large because of the different clinical conditions in each institution, different types of cases, systems, TPS, and 
QA tools (16). As the number of verification plans and parameters increases, the necessity to establish suitable and common MLC parameters increases accordingly. The effort to optimize MLC parameters is large in terms of accomplishing comprehensive system commissioning in a limited time frame. Additionally, Akino et al. have shown that the variation in dosimetric characteristics for small field sizes, especially for $5 \times 5 \mathrm{~mm}^{2}$ field sizes, depends on the respective detectors and not on the treatment devices used (17).

Several reports have focused on multi-institutional measurements of IMRT or/and VMAT. However, few reports have considered the variance of patient-specific QA including the variability of beam data and MLC parameters with the same plan and the same type of QA tools $(18,19)$. Therefore, this study aimed to investigate the potential differences between multi-institutional measurements and TPS calculation as modeled by RBD for patient-specific QA, focusing on the variability of beam data and MLC parameters acquired using the same plan and the same type of QA tools.

\section{Patients and Methods}

Data collection. We acquired data from 11 TrueBeam in 9 different institutions employing Eclipse TPSs. The scanning data, PDD, and the OCR at a depth of $10 \mathrm{~cm}\left(\mathrm{~d}_{10}\right)$ and a depth of maximum dose $\left(\mathrm{d}_{\max }\right)$ in crossline were collected. Moreover, data measured in $10 \mathrm{MV}, 30 \times 30,100 \times 100$, and $300 \times 300 \mathrm{~mm}^{2}$ field sizes using a CC13 (IBA Dosimetry GmbH, Schwarzenbruck, Germany) or a Semiflex (PTW, Freiburg, Germany) ionization chamber were extracted to compare them with the RBD data collected from the CC13. The PDD data were resampled with 1 $\mathrm{mm}$ interval and were normalized at $\mathrm{d}_{\max }$. In addition, the OCR data were resampled with $1 \mathrm{~mm}$ interval and the calculated position of the center of the full width was corrected at half maximum to focus on the shape of the OCR profile. The OCR data were also normalized at the center-axis values. The dose difference (DD) from RBD at the exponential region and $\mathrm{PDD}_{10}$ were used to investigate the variation of the PDD data. In contrast, the full width half maximum (FWHM) and the penumbra width were used to investigate the variation of the OCR data. The OCR penumbra region was defined by the positions of the $20 \%-80 \%$ profile, and the penumbra width was then defined as the width of the penumbra region. The Akilles RT (RADLab Inc., Osaka, Japan) software was used to analyze the scanning data. In addition, DLG measurement values and the transmission factor for $10 \mathrm{MV}$ were collected. Their average and SD values were used for the subsequent analysis of MLC variations.

\section{VMAT verification}

Verification plan creation. The Eclipse (ver. 15.0.1) system was used to develop a VMAT plan for institutional verification, in which the scanning data were modeled by RBD. In addition, couch and MLC parameters were modeled based on the values provided by the Osaka University affiliated hospital because of their enhanced beam conformance (EBC) option. TrueBeam with EBC option can be adjusted so that the energies are within the tolerance
Table I. Dose constraints to organs at risk used in the Osaka University Hospital.

\begin{tabular}{llll}
\hline Structure & \multicolumn{3}{c}{ Dose constraint } \\
\hline \multirow{2}{*}{ Rectum } & Dmax & $\leq$ & $105 \%$ \\
& $\mathrm{~V} 90 \%$ & $\leq$ & $10 \%$ \\
& $\mathrm{~V} 80 \%$ & $\leq$ & $15 \%$ \\
& $\mathrm{~V} 70 \%$ & $\leq$ & $25 \%$ \\
& $\mathrm{~V} 50 \%$ & $\leq$ & $50 \%$ \\
Bladder & $\mathrm{Dmax}$ & $\leq$ & $105 \%$ \\
& $\mathrm{~V} 90 \%$ & $\leq$ & $20 \%$ \\
& $\mathrm{~V} 80 \%$ & $\leq$ & $30 \%$ \\
& $\mathrm{~V} 70 \%$ & $\leq$ & $40 \%$ \\
\hline
\end{tabular}

level before transporting them to the institution, and a narrow tolerance level was selected in TrueBeam setting. This plan was calculated with the American Association of Physicists in Medicine (AAPM) Task Group (TG)-119 prostate structures (20) on the I'mRT phantom (IBA Dosimetry) computed tomography (CT) scan data according to the Osaka University Hospital dose constraint in 2018. The CT value of the phantom was replaced with water in the dose calculation. The prescription dose was 78 Gy in 39 fractions delivered to the reference point in the PTV, with $\geq 95 \%$ of the PTV covered by $95 \%$ of the prescribed dose, and the maximum dose was $\leq 107 \%$ of the prescribed dose (i.e. with $10 \mathrm{MV}$ in two full arcs). Dose constraints to the organs at risk are listed in Table I. Fifty percent isodose line did not exceed the entire rectum. The results of patient-specific QA were investigated in accordance with the chamber's point dose and the film's dose distribution.

Point dose. The point dose at the center of the I'mRT phantom with VMAT plan was calculated in Eclipse at our Lab and was compared with the measured point dose obtained by a CC13 or a Semiflex ionization chamber, using cross-calibrations followed by AAPM TG119 (20). For cross-calibration, the point dose at the center of the I'mRT phantom was measured by a $100 \mathrm{MU}$ irradiation for a $10 \times 10$ $\mathrm{cm}^{2}$ defined field size of $10 \mathrm{MV}$ at $90^{\circ}$ and $270^{\circ}$ gantry angles, respectively, using ionization chamber. Furthermore, the dose at 9$\mathrm{cm}$ depths and for a $100 \mathrm{MU} 10 \times 10 \mathrm{~cm}^{2}$ field size of $10 \mathrm{MV}$ was calculated at virtual water in the Eclipse system at each institution. This was performed in order to investigate potential simple output differences in each institution. The DD was then assessed as the difference between the measured and the calculated doses.

Dose distribution. GAFCHROMIC EBT3 films (Ashland corporation, Qayne, $\mathrm{NJ}$ ) were used for the acquisition of measurements from the same box (Lot\#: 09071701) and were analyzed by the Akilles RT software. The dose response curve was acquired by 10 distinct film pieces $(0,24.2,48.4,72.5,96.7,145.1$, $193.4,241.8,290.1$, and $386.8 \mathrm{cGy}$ ) that were cut into the shape of a $3.2-\mathrm{cm}$ square. VMAT plan dose distribution was measured at the center of the I'mRT phantom sagittal and coronal axes using films cut to a size of about $12.7 \times 4.2 \mathrm{~cm}^{2}$. All films were scanned 1 week after exposure by a flatbed scanner GT-X980 (Seiko EPSON Corp., Nagano, Japan) and its associated software, EPSON SCAN (ver. 3.9.3.2 JA) (Seiko EPSON Corp.). Scanner settings were set in 
(a) Field Size: $30 \times 30 \mathrm{~mm}^{2}$

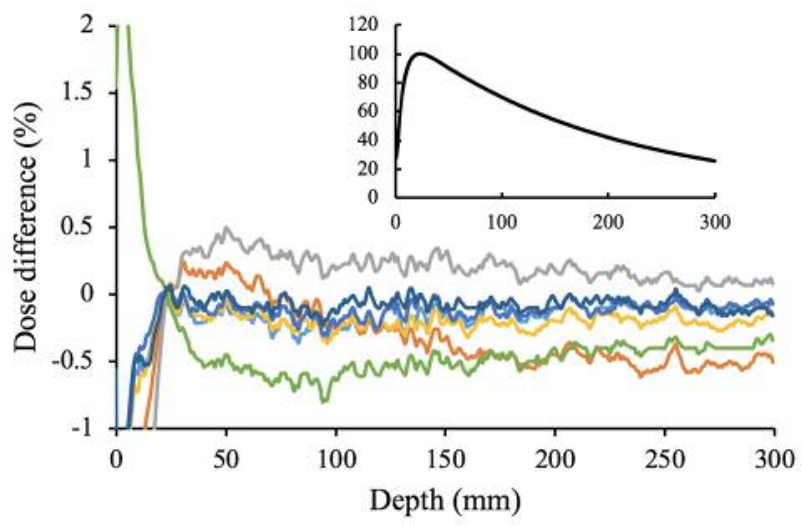

(b) Field Size: $100 \times 100 \mathrm{~mm}^{2}$

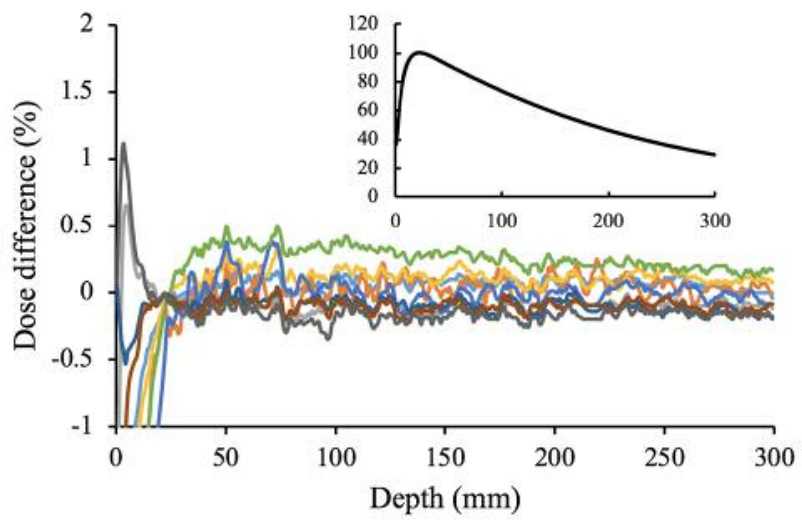

(c) Field Size: $300 \times 300 \mathrm{~mm}^{2}$

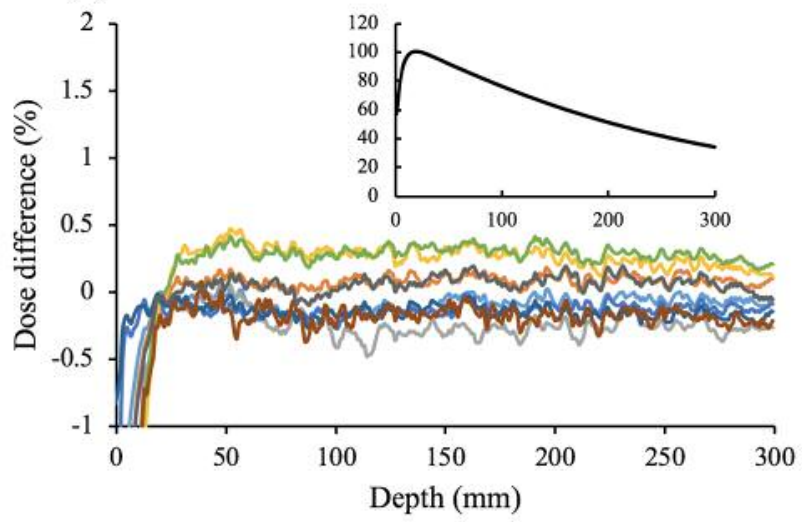

Figure 1. Percentage depth dose curve of representative beam data $(R B D)$ and dose difference between each curve and $R B D$ curve for $(a)$ $30 \times 30$, (b) $100 \times 100$, and (c) $300 \times 300 \mathrm{~mm}^{2}$ field size.

transmission mode with 48 bits color TIFF image, scan resolution of 72 dots per inch (dpi), and professional mode, and without using all available image correction modes. All films were positioned so that the long axis of the uncut film was parallel to that of the scanner. (a) Field Size: $30 \times 30 \mathrm{~mm}^{2}$

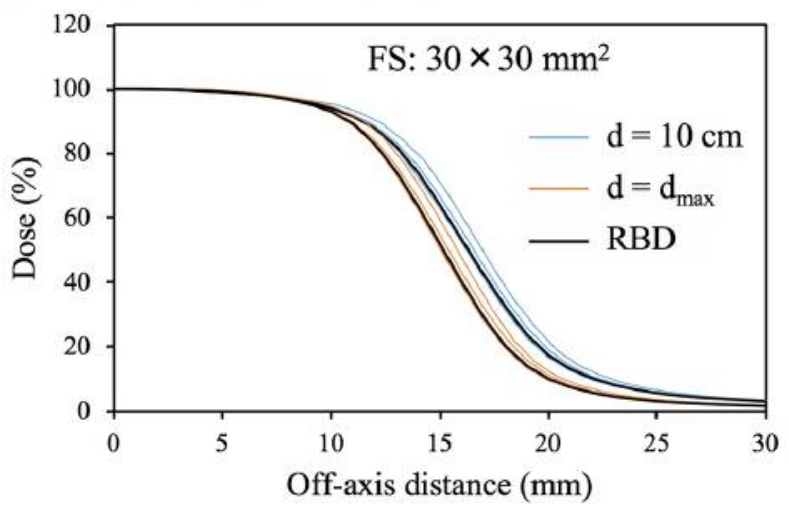

(b) Field Size: $100 \times 100 \mathrm{~mm}^{2}$

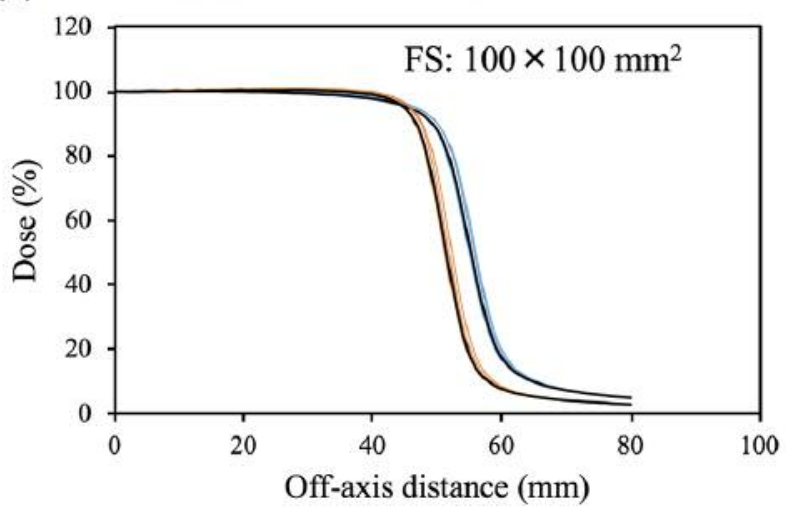

(c) Field Size: $300 \times 300 \mathrm{~mm}^{2}$

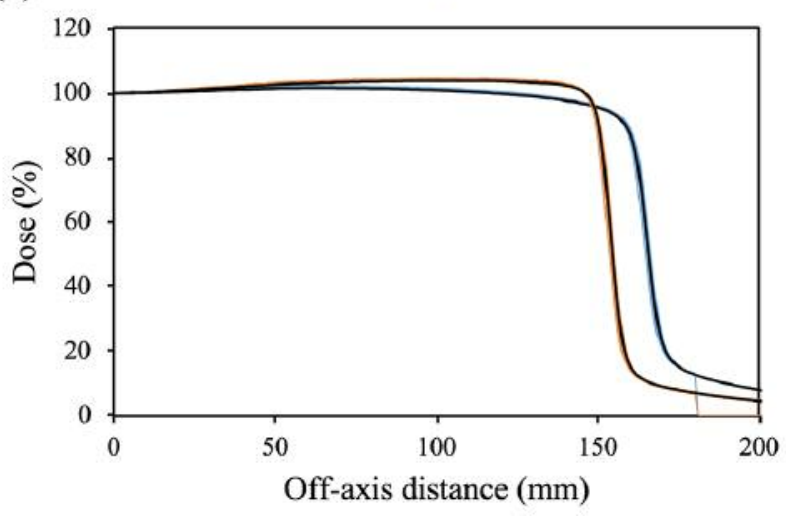

Figure 2. Off-center ratio (OCR) profiles for (a) 30×30, (b) $100 \times 100$, and (c) $300 \times 300 \mathrm{~mm}^{2}$. The black line represents the OCR curve of representative beam data. The blue lines and the red lines represent the data for $d_{10}$ and for $d_{\text {max }}$, respectively.

Seven pieces of films $(0,48,4,96.7,145.1,193.4,241.8$, and 386.8 cGy) used for the acquisition of dose response curves were scanned at 19 points in the short axis using a $3-\mathrm{cm}$ step and were subsequently used for scanner-calibration and to correct potential 
Table II. Analysis of percentage depth dose (PDD) and off-center ratio (OCR) measured by CC13 or Semiflex ionization chamber. Results are presented as the average $\pm S D$ and the range of difference is acquired by subtracting each data measurements from the representative beam data $(R B D)$ ones.

\begin{tabular}{|c|c|c|c|c|c|}
\hline & Field size $\left(\mathrm{mm}^{2}\right)$ & Depth & $\mathrm{N}$ & Average $\pm \mathrm{SD}$ & $\begin{array}{l}\text { Range of difference } \\
\text { (RBD-each data) }\end{array}$ \\
\hline \multirow[t]{3}{*}{ PDD10 (\%) } & $30 \times 30$ & \multirow[t]{3}{*}{$10 \mathrm{~cm}$} & 7 & $69.7 \pm 0.2$ & $-0.2 \sim 0.6$ \\
\hline & $100 \times 100$ & & 9 & $73.5 \pm 0.2$ & $-0.3 \sim 0.2$ \\
\hline & $300 \times 300$ & & 9 & $75.8 \pm 0.2$ & $-0.3 \sim 0.5$ \\
\hline \multirow[t]{6}{*}{ OCR FWHM (mm) } & \multirow{2}{*}{$30 \times 30$} & $10 \mathrm{~cm}$ & \multirow[t]{2}{*}{4} & $32.8 \pm 0.8$ & $-1.2 \sim 0.6$ \\
\hline & & $\mathrm{d}_{\max }$ & & $30.5 \pm 0.8$ & $-1.2 \sim 0.5$ \\
\hline & \multirow[t]{2}{*}{$100 \times 100$} & $10 \mathrm{~cm}$ & \multirow[t]{2}{*}{6} & $110.7 \pm 0.7$ & $-1.1 \sim 0.7$ \\
\hline & & $\mathrm{d}_{\max }$ & & $103.0 \pm 0.7$ & $-1.0 \sim 0.7$ \\
\hline & \multirow{2}{*}{$300 \times 300$} & $10 \mathrm{~cm}$ & \multirow[t]{2}{*}{9} & $331.1 \pm 1.0$ & $-1.1 \sim 2.1$ \\
\hline & & $\mathrm{d}_{\max }$ & & $308.6 \pm 1.0$ & $-1.0 \sim 2.0$ \\
\hline \multirow[t]{6}{*}{ OCR penumbra width $(\mathrm{mm})$} & \multirow[t]{2}{*}{$30 \times 30$} & $10 \mathrm{~cm}$ & \multirow[t]{2}{*}{4} & $6.3 \pm 0.1$ & $0.0 \sim 0.2$ \\
\hline & & $\mathrm{d}_{\max }$ & & $5.8 \pm 0.1$ & $-0.1 \sim 0.0$ \\
\hline & \multirow[t]{2}{*}{$100 \times 100$} & $10 \mathrm{~cm}$ & \multirow[t]{2}{*}{6} & $7.4 \pm 0.1$ & $-0.1 \sim 0.2$ \\
\hline & & $\mathrm{d}_{\max }$ & & $6.2 \pm 0.1$ & $-0.1 \sim 0.0$ \\
\hline & \multirow[t]{2}{*}{$300 \times 300$} & $10 \mathrm{~cm}$ & \multirow[t]{2}{*}{9} & $9.0 \pm 0.1$ & $-0.1 \sim 0.3$ \\
\hline & & $\mathrm{d}_{\max }$ & & $6.6 \pm 0.1$ & $-0.2 \sim 0.2$ \\
\hline
\end{tabular}

inhomogeneities in the short axis direction. The pixel value of the non-irradiated film was changed over time, and this change affected the acquired results of dose evaluation. Therefore, irradiated films used in VMAT plan were simultaneously corrected by non-irradiated films. The corrected films were then compared with the planned gamma pass rate dose distribution with $2 \% / 2 \mathrm{~mm}$ and $3 \% / 2 \mathrm{~mm}$ criteria, and a $10 \%$ threshold. Dose distribution measurements were performed in each institution within 1 month following the acquisition of the dose response curve at the Osaka University Hospital to minimize the influence of time-dependency on films.

\section{Results}

Scanning data. The DD of PDD between the measurement data in each institution and the RBD are shown in Figure 1, and the OCR profile curves including the RBD are shown in Figure 2. The analysis of scanning data results, the average \pm SD values, and the range of difference acquired by subtracting each data measurements from the RBD ones are shown in Table II. In the exponential regions, the DD values of PDD with $30 \times 30$, $100 \times 100$, and $300 \times 300 \mathrm{~mm}^{2}$ field sizes were $\leq 1.0 \%, \leq 0.5 \%$, and $\leq 0.5 \%$, respectively. The SD of PDD ${ }_{10}$, OCR FWHM, and OCR penumbra width were $\leq 0.2 \%, \leq 1.0 \mathrm{~mm}$, and $\leq 0.1 \mathrm{~mm}$ for all field sizes, respectively. The range of difference between the RBD and PDD ${ }_{10}$, OCR FWHM, and OCR penumbra width data from each institution were $\leq 0.6 \%, \leq 2.1 \mathrm{~mm}$, and $\leq 0.3 \mathrm{~mm}$ for all field sizes, respectively.

MLC parameters. Table III shows the average and SD values of the measured MLC parameters. The SD of the measured DLG values was $0.14 \mathrm{~mm}$. The SD of the measured transmission factor was $0.07 \%$.
Table III. Average $\pm S D$ of measured multi-leaf collimator (MLC) parameters.

\begin{tabular}{lccc}
\hline $\begin{array}{l}\text { Measured } \\
\text { MLC parameter }\end{array}$ & $\mathrm{N}$ & Average & SD \\
\hline DLG (mm) & 10 & 1.26 & 0.14 \\
Transmission factor $(\%)$ & 10 & 1.72 & 0.07 \\
\hline
\end{tabular}

Table IV. Average $\pm S D$ of the dose error between the planned and the measured doses for volumetric arc therapy (VMAT) verification and average $\pm S D$ of the dose calculated by treatment planning system for $100 \mathrm{MU}$ irradiation.

\begin{tabular}{lccc}
\hline & $\mathrm{N}$ & Average & SD \\
\hline VMAT dose error (\%) & 11 & 1.0 & 0.6 \\
100MU planned dose (cGy) & 11 & 86.5 & 0.4
\end{tabular}

Point dose. Table IV exhibits dose error results between the planned and the measured doses for VMAT verification, and the variation of output was calculated by the Eclipse system using a simple irradiation for $100 \mathrm{MU}$. The VMAT dose error was $<2 \%$ in all institutions with an SD of $0.6 \%$. The average planned dose for $100 \mathrm{MU}$ was $86.5 \mathrm{cGy}$, and the SD was $0.4 \mathrm{cGy}$.

Dose distribution. Figure 3 illustrates the typical gamma analysis results with $2 \% / 2 \mathrm{~mm}$ criteria that compare the dose distribution measured with film with that calculated by the TPS 
(a) Coronal plane

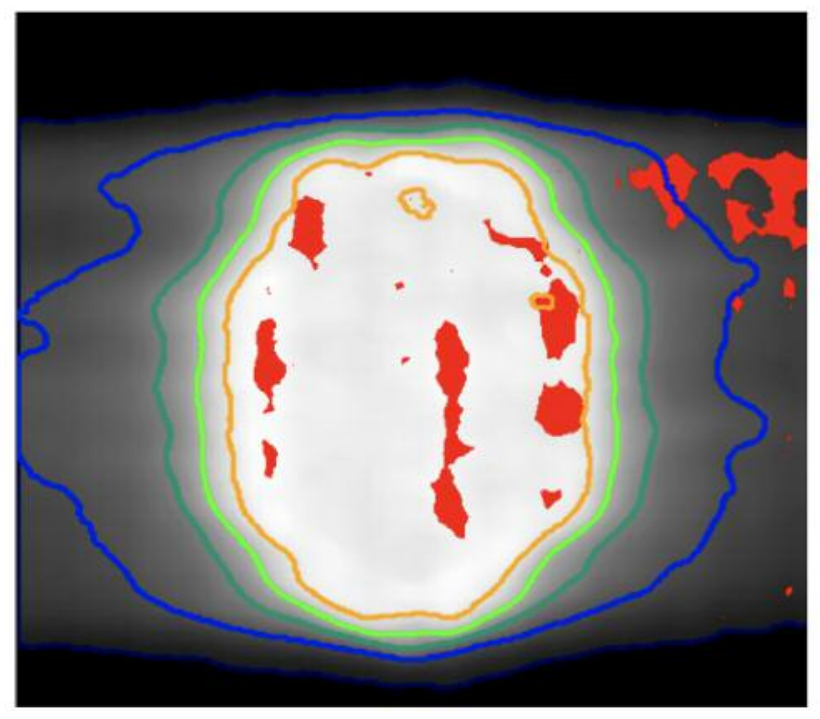

(b) Sagittal plane

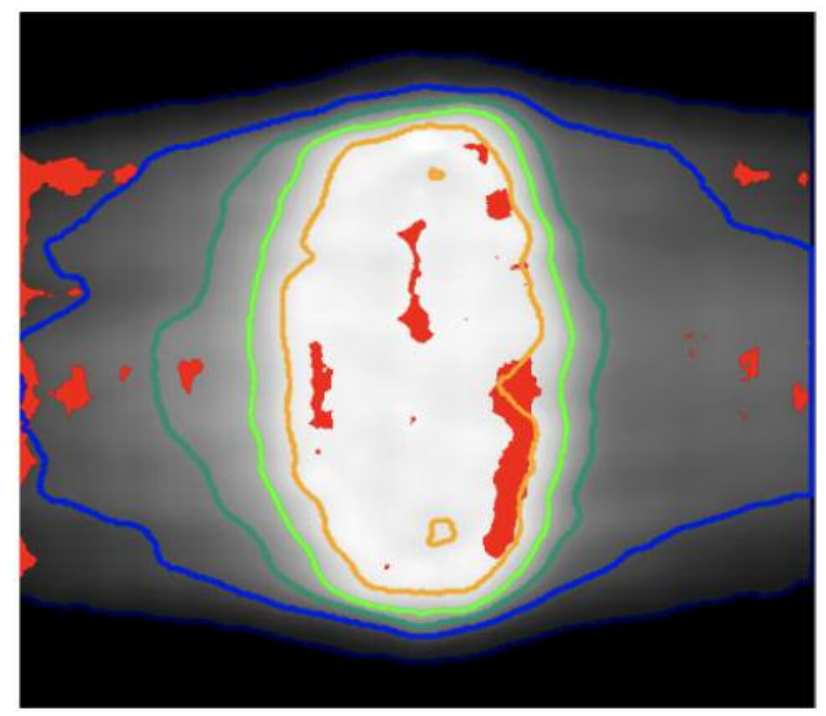

Figure 3. Typical results of gamma analysis comparing the dose distribution measured with film with that calculated by treatment planning system in (a) coronal plane, and in (b) sagittal plane. Red pixels indicate a gamma value greater than 1. Orange, light green, green, blue, and dark blue lines indicate isodose lines for $90 \%, 70 \%, 50 \%, 30 \%$, and $10 \%$ of predicted dose, respectively.

Eclipse. This comparison underlines the presence of failure points both inside and outside the PTV. The variation of gamma pass rates is shown in Table $\mathrm{V}$. The number of institutions with gamma pass rates below $95 \%$ was three for $2 \% / 2 \mathrm{~mm}$ gamma criteria and zero for $3 \% / 2 \mathrm{~mm}$ gamma criteria, for both planes. Additionally, the average pass rates for $2 \% / 2 \mathrm{~mm}$ gamma criteria and $3 \% / 2 \mathrm{~mm}$ gamma criteria exceeded $96 \%$ and $99 \%$, respectively. In addition, the SD values of gamma pass rates for $2 \% / 2 \mathrm{~mm}$ gamma criteria and $3 \% / 2 \mathrm{~mm}$ gamma criteria were within $3.0 \%$ and $1.0 \%$, respectively.

\section{Discussion}

In this study, the difference between multi-institutional measurements and TPS calculations modeled by RBD for patient-specific QA of prostate VMAT plan was evaluated by considering the PDD, OCR, and MLC parameters. This is because the number of reports investigating the multiinstitutional accuracy of RBD modeling in the same prostate VMAT plan are limited.

In this study, 11 TrueBeam in 9 different institutions that employ Eclipse TPSs were evaluated. This evaluation was performed according to our previous report (5), and involved the analysis of average scanning data across 21 TrueBeam and RBD for PDD, OCR, and OPF for field sizes of $30 \times 30$, $100 \times 100$, and $300 \times 300 \mathrm{~mm}^{2}$. Almost all acquired data were in the same range as in the previous report. $\mathrm{PDD}_{10}$ variations shown in Table II were small and similar to the findings
Table V. Average $\pm S D$ of gamma pass rate.

\begin{tabular}{lcccc}
\hline & Plane & $\mathrm{N}$ & Average & $\mathrm{SD}$ \\
\hline $2 \% / 2 \mathrm{~mm} \gamma$ pass rate $(\%)$ & Cor & 11 & 96.0 & 3.0 \\
& Sag & 11 & 96.2 & 2.6 \\
$3 \% / 2 \mathrm{~mm} \gamma$ pass rate $(\%)$ & Cor & 11 & 99.1 & 0.9 \\
& Sag & 11 & 99.2 & 1.0 \\
\hline
\end{tabular}

reported by Chang et al., and Glide-Hurst et al. $(1,3)$, in which data were measured with a CC13 ionization chamber. Chang et al. (1) evaluated the commissioning data of three TrueBeam machines and reported that their PDD and beam profiles were consistent. Furthermore, Glide-Hurst et al.(3) reported that the measured $\mathrm{PDD}_{10}$ variation in their study was $<0.3 \%$. The variation of penumbra width of OCR at $\mathrm{d}_{10}$ was also small and similar to the results presented by Glide-Hurst et al. (3): the average penumbra width and the SD at $\mathrm{d}_{10}$ were $7.4 \pm 0.1 \mathrm{~mm}$ and $9.1 \pm 0.2 \mathrm{~mm}$ for $10 \times 10 \mathrm{~cm}^{2}$ and $30 \times 30 \mathrm{~cm}^{2}$ fields, respectively. Other results of scanning data analysis, e.g., OCR FWHM, also exhibited a small variation because their SD values were small compared with their average values. Therefore, and according to these results, the data collected in this study exhibited a small variation.

The variability between point dose for VMAT irradiation and point dose for 100MU simple irradiation was similar. The point DD of VMAT verification was $<2 \%$ in all institutions. 
VMAT uses a very complex planning method. However, our results highlight that there is a very small variation in the dose delivered to patients (similar to a simple irradiation). Dose distribution analysis was consistent even in highly modulated areas affected by the MLC parameters (21). However, the SD value of $2 \% / 2 \mathrm{~mm}$ gamma analysis was $3 \%$, whereas that of $3 \% / 2 \mathrm{~mm}$ gamma analysis was $1 \%$. Additionally, the minimum gamma pass rate with $2 \% / 2 \mathrm{~mm}$ criteria was $90.3 \%$, whereas the maximum gamma pass rate following these criteria was $99.1 \%$, although all results were within the tolerance rates indicated by AAPM TG-218 (21). In contrast, the minimum gamma pass rate with $3 \% / 2 \mathrm{~mm}$ criteria was $97.1 \%$. These results revealed that the dose difference of many institutions ranged from $2 \%$ to $3 \%$. These results could be the important starting point to consider whether RBD modeling should be adopted in each institution.

Variations of measured MLC parameters were small; the SD of DLG and transmission factor were $0.14 \mathrm{~mm}$ and $0.1 \%$, respectively. In our previous report, the median and the SD of DLG and transmission factor of 18 TrueBeam at $10 \mathrm{MV}$ were $1.32 \mathrm{~mm}, 0.21 \mathrm{~mm}$ and $1.72 \%, 0.06 \%$, respectively. Conversely, the median and $95 \%$ confidence interval of the modified DLG values, and MLC leaf transmission factors for TrueBeam with Millennium120 MLC were $1.93 \mathrm{~mm}(1.38-2.48)$ and $1.78 \%$ (1.39-2.17), respectively. The optimized MLC parameters, especially the DLG one, depended on the characteristics of the treatment plans developed in each institution (13), such as the irradiation method, case, and target, among others. In general, medical physicists need a lot of time to identify the optimum MLC parameters. VMAT verification measurement in this study was in good agreement with the results of the Eclipse modeled by RBD and with the specific MLC parameters-2.60 mm of DLG and $1.79 \%$ of transmission factor. Therefore, we suggest that provided that the planning policy is the same in each institution, modeling common MLC parameters can be very effective, and thus, a certain level of accuracy can be achieved to initiate treatment. However, and as mentioned earlier, the minimum gamma pass rate with $2 \% / 2 \mathrm{~mm}$ criteria was $90.3 \%$. Consequently, it is necessary to be extremely careful when modeling common MLC parameters in institutions where control VMAT is within $2 \% / 2 \mathrm{~mm}$ tolerance. Similar attention is needed in case of RBD modeling.

Commissioning for IMRT and VMAT is a time-consuming process that requires significant effort. Molineu et al. reported errors exceeding tolerance in $18.4 \%$ institutions when comparing measured and planned values in head and neck IMRT (18). Regardless of how efficiently a plan is developed, it can be of no importance if the TPS accuracy is poor. However, RBD modeling with MLC parameters could help physicists in reducing efforts for commissioning with safety and within accepted tolerance values defined by the respective guidelines. In such cases, the adoption of common MLC parameters, can facilitate the development of effective plans in conventional radiotherapy but also in VMAT with an instant accuracy of prediction.

\section{Conclusion}

This study verifies the irradiation accuracy of the same VMAT plan in multiple institutions. The variation of equipment used in this study was very small and the impact of complex VMAT on the dosimetric accuracy was less because the point dose and dose distribution were consistent with the planned values. These results suggest that common MLC parameters could be set on TPS between institutions with the same planning policy.

\section{Conflicts of Interest}

The 3rd author, YA, is a developer of the commercial software Akilles RT, which was used for analysis in this study.

\section{Authors' Contributions}

Goto Hironao: all sections; Hirokazu Mizuno: all sections; Yuichi Akino, Masaru Isono, Yoshihiro Tanaka, Norihisa Masai, Toshijiro Yamamoto, Masahiko Koizumi: data collection.

\section{Acknowledgements}

The Authors acknowledge the support of Katarou-Kai, whose contribution made this work possible. This work was supported by Fostering Health Professionals for Changing Needs of Cancer.

\section{References}

1 Chang Z, Wu Q, Adamson J, Ren L, Bowsher J, Yan H, Thomas $A$ and Yin FF: Commissioning and dosimetric characteristics of TrueBeam system: composite data of three TrueBeam machines. Med Phys 39(11): 6981-7018, 2012. PMID: 23127092. DOI: 10.1118/1.4762682

2 Beyer GP: Commissioning measurements for photon beam data on three TrueBeam linear accelerators, and comparison with Trilogy and Clinac 2100 linear accelerators. J Appl Clin Med Phys 14(1): 273-288, 2013. PMID: 23318395. DOI: 10.1120/jacmp.v14i1.4077

3 Glide-Hurst C, Bellon M, Foster R, Altunbas C, Speiser M, Altman M, Westerly D, Wen N, Zhao B, Miften M, Chetty IJ and Solberg T: Commissioning of the Varian TrueBeam linear accelerator: a multi-institutional study. Med Phys 40: 031719, 2013. PMID: 23464314. DOI: 10.1118/1.4790563

4 Kerns JR, Followill DS, Lowenstein J, Molineu A, Alvarez P, Taylor PA, Stingo FC and Kry SF: Technical Report: Reference photon dosimetry data for Varian accelerators based on IROCHouston site visit data. Med Phys 43(5): 2374, 2016. PMID: 27147349. DOI: $10.1118 / 1.4945697$

5 Tanaka Y, Mizuno H, Akino Y, Isono M, Masai N and Yamamoto T: Do the representative beam data for TrueBeam linear accelerators represent average data? J Appl Clin Med Phys 20: 51-62, 2019. PMID: 30636358. DOI: 10.1002/acm2.12518 
6 Chui CS, Chan MF, Yorke E, Spirou S and Ling CC: Delivery of intensity-modulated radiation therapy with a conventional multileaf collimator: comparison of dynamic and segmental methods. Med Phys 28(12): 2441-2449, 2001. PMID: 11797947. DOI: $10.1118 / 1.1418018$

7 Essers M, Langen Md, Dirkx MLP and Heijmen BJ: Commissioning of a commercially available system for intensity-modulated radiotherapy dose delivery with dynamic multileaf collimation. Radiother Oncol 60: 215-224, 2001. PMID: 11439216. DOI: 10.1016/s0167-8140(01)00317-6

8 Otto K: Volumetric modulated arc therapy: IMRT in a single gantry arc. Med Phys 35(1): 310-317, 2008. PMID: 18293586. DOI: $10.1118 / 1.2818738$

9 Palma D, Vollans E, James K, Nakano S, Moiseenko V, Shaffer $\mathrm{R}$, McKenzie M, Morris J and Otto K: Volumetric modulated arc therapy for delivery of prostate radiotherapy: comparison with intensity-modulated radiotherapy and three-dimensional conformal radiotherapy. Int J Radiat Oncol Biol Phys 72(4): 996-1001, 2008. PMID: 18455326. DOI: 10.1016/j.ijrobp.2008.02.047

10 Rangel A and Dunscombe P: Tolerances on MLC leaf position accuracy for IMRT delivery with a dynamic MLC. Med Phys 36(7): 3304-3309, 2009. PMID: 19673226. DOI: 10.1118/ 1.3134244

11 LoSasso T, Chui C-S and Ling CC: Physical and dosimetric aspects of a multileaf collimation system used in the dynamic mode for implementing intensity modulated radiotherapy. Med Phys 25(10): 1919-1927, 1998. PMID: 9800699. DOI: $10.1118 / 1.598381$

12 Kielar KN, Mok E, Hsu A, Wang L and Luxton G: Verification of dosimetric accuracy on the TrueBeam STx: rounded leaf effect of the high definition MLC. Med Phys 39(10): 6360-6371, 2012. PMID: 23039672. DOI: 10.1118/1.4752444

13 Middlebrook ND, Sutherland B and Kairn T: Optimization of the dosimetric leaf gap for use in planning VMAT treatments of spine SABR cases. J Appl Clin Med Phys 18(4): 133-139, 2017. PMID: 28574219. DOI: $10.1002 / \mathrm{acm} 2.12106$

14 Chauvet I, Petitfils A, Lehobey C, Kristner JY, Brunet Y, Lembrez R, Gaboriaud G, Mazal A, Zefkili S and Rosenwald JC: The sliding slit test for dynamic IMRT: a useful tool for adjustment of MLC related parameters. Phys Med Biol 50(4): 563-580, 2005. PMID: 15773620. DOI: 10.1088/0031-9155/50/4/001
15 Szpala S, Cao F and Kohli K: On using the dosimetric leaf gap to model the rounded leaf ends in VMAT/RapidArc plans. J Appl Clin Med Phys 15(2): 67-84, 2014. PMID: 24710433. DOI: 10.1120/jacmp.v15i2.4484

16 Isono M, Akino Y, Mizuno H, Tanaka Y, Masai N and Yamamoto $\mathrm{T}$ : Inter-unit variability of multi-leaf collimator parameters for IMRT and VMAT treatment planning: a multi-institutional survey. J Radiat Res 10: 1093, 2020. PMID: 31927580. DOI: $10.1093 / \mathrm{jrr} / \mathrm{rrz} 082$

17 Akino $\mathrm{Y}$, Mizuno $\mathrm{H}$, Tanaka $\mathrm{Y}$, Isono $\mathrm{M}$, Masai $\mathrm{N}$ and Yamamoto T: Inter-institutional variability of small-fielddosimetry beams among HD120 ${ }^{\mathrm{TM}}$ multileaf collimators: a multi-institutional analysis. Phys Med Biol 63: 205018, 2018. PMID: 30255847. DOI: 10.1088/1361-6560/aae450

18 Molineu A, Hernandez N, Nguyen T, Ibbott G and Followill D: Credentialing results from IMRT irradiations of an anthropomorphic head and neck phantom. Med Phys 40(2): 022101, 2013. PMID: 23387762. DOI: 10.1118/1.4773309

19 Clark CH, Hussein M, Tsang Y, Thomas R, Wilkinson D, Bass G, Snaith J, Gouldstone C, Bolton S, Nutbrown R, Venables K and Nisbet A: A multi-institutional dosimetry audit of rotational intensity-modulated radiotherapy. Radiother Oncol 113(2): 272278, 2014. PMID: 25465734. DOI: 10.1016/j.radonc.2014.11.015

20 Ezzell GA, Burmeister JW, Dogan N, LoSasso TJ, Mechalakos JG, Mihailidis D, Molineu A, Palta JR, Ramsey CR, Salter BJ, Shi J, Xia P, Yue NJ and Xiao Y: IMRT commissioning: multiple institution planning and dosimetry comparisons, a report from AAPM Task Group 119. Med Phys 36(11): 5359-5373, 2009. PMID: 19994544. DOI: 10.1118/1.3238104

21 Miften M, Olch A, Mihailidis D, Moran J, Pawlicki T, Molineu A, Li H, Wijesooriya K, Shi J, Xia P, Papanikolaou N and Low DA: Tolerance limits and methodologies for IMRT measurement-based verification QA: Recommendations of AAPM Task Group No. 218. Med Phys 45(4): e53-e83, 2018. PMID: 29443390. DOI: $10.1002 / \mathrm{mp} .12810$

Received January 27, 2020

Revised February 13, 2020

Accepted February 14, 2020 